\title{
Des innovations technologiques au monde post- apocalyptique. Enseignements tirés des films de science-fiction.
}

\author{
Technological innovations in the post-apocalyptic world. Lessons learned \\ from science fiction movies.
}

\author{
Nadine Boudou ${ }^{1}$ \\ ${ }^{1}$ Prof. de philosophie, chercheuse indépendante rattachée au LERSEM (Montpellier), nadine.boudou@orange.fr
}

\begin{abstract}
RÉSUMÉ. L'objectif de cet article est d'analyser les contradictions que relèvent de nombreux films de science-fiction au sujet de notre mode de développement et de ses dysfonctionnements. Ces contradictions, dont ces œuvres rendent compte, nous semblent dues à l'incompatibilité entre des innovations présumées infinies et le caractère fini de notre monde. Malgré l'infinité des possibles que les innovations technologiques nous promettent, ces récits en montrent aussi leurs limites et leur dangerosité. Ce qui nous permettra de montrer quels enseignements nous pouvons tirer de ces récits dans lesquels les innovations technologiques sont néfastes à l'humanité et à son environnement.

ABSTRACT. The objective of this article is to analyze the contradictions between our future vision about world development and a possible apocalyptic end, a subject of various science fiction movies. These contradictions seem to us due to the incompatibility between the presumed infinite innovations and finite resources of our planet. In spite of the infinite opportunities which technological innovations promise us, these narratives demonstrate their limits and their risks. These science fiction movies act as a real warning system, revealing the possible poisonous consequences of some technological innovation trends that could be fatal for humanity and its environment.

MOTS-CLÉS. Culture technique, imaginaire, ingénieur, innovation, science-fiction.

KEYWORDS. Technical culture, imaginary, engineer, innovation, science fiction.
\end{abstract}

\section{Introduction}

La science-fiction met en intrigue un monde dans lequel la réalité matérielle et la vie sociale sont bouleversées par les innovations technologiques. Ces transformations se caractérisent par de telles avancées qu'elles permettraient de soulager les humains d'un grand nombre de tâches jusqu'à les délivrer de contraintes attachées à leur condition. L'humanité en plus d'être délivrée de tout effort physique et intellectuel n'aurait plus à endurer la maladie et la mort grâce à des thérapies lui assurant la santé, la longévité voire l'immortalité. Capable d'échapper aux limites de son corps et de son esprit, elle pourrait aussi échapper aux limites du temps et de l'espace en quittant la Terre. Tels sont les apports que certaines innovations promettent et que de nombreux récits de science-fiction développent. Pourtant, malgré le haut niveau de technologie auquel l'humanité serait parvenue, ces scénarios ne cessent de rendre compte de situations catastrophiques comme conséquences directes ou indirectes de ces innovations. L'idéal de progrès basé sur une croissance illimitée et sur des innovations infinies est contredit par l'effondrement de la civilisation. Les progrès que ces innovations semblent contenir sont limités par les conséquences apocalyptiques ou post-apocalyptiques décrites dans ces fictions.

Alors que les films apocalyptiques envisagent l'avant ou le moment de la catastrophe, les films postapocalyptiques représentent le monde de l'après catastrophe. Ces fictions ont toutes pour point commun une interrogation sur le devenir de la Terre et de l'humanité en cas d'effondrement de la civilisation. La Terre ayant perdu son caractère d'habitabilité, l'humanité est privée des systèmes de protection et d'organisation qui encadraient et régulaient ses activités. Ces fictions peuvent, par contre, se différencier sur les causes de la catastrophe: domination des machines, pollution, pandémie, 
changement climatique, raréfaction des ressources. Malgré ces singularités, les films apocalyptiques et post-apocalyptiques obéissent à un même schéma directeur qui donne, à ce sous-genre de la sciencefiction, son homogénéité. Les enseignements que nous pouvons tirer de ces films seront accompagnés, en parallèle, de ceux que nous apportent les analyses de certains chercheurs qui insistent sur la nécessité de respecter les limites planétaires pour les générations présentes et futures.

Nous verrons en un premier temps comment la science-fiction normalise l'idée d'un monde révolutionné par l'usage du numérique et construit la vision d'un monde dominé par des machines douées d'autonomie. Cependant, malgré la description des possibilités offertes par ces innovations, nous montrerons que de nombreux films s'intéressent à ce qui peut résulter de catastrophique pour nos sociétés. Ce qui nous permettra d'analyser à quoi ces films de science-fiction peuvent servir dans la mesure où, s'ils nous laissent imaginer certains possibles, ils en montrent tout autant leur dangerosité que leur caractère non viable sur le long terme. Malgré le caractère catastrophiste de ces films nous montrerons, néanmoins, qu'ils peuvent aussi avoir un impact positif sur le public.

\section{Le devenir-machine de l'humanité}

La série télévisée suédoise, Real Humans (Lars Lundström, 2012-2014), pose certaines des questions que peut soulever l'intrusion de robots dans les sociétés humaines. Cette série développe sur deux saisons des interrogations sur la définition de l'humain et sur ce qui le différencie de la machine. L'histoire oppose des humains à des hubots, contraction d'humain et de robot, pouvant être des robots humanoïdes, des clones ou des hommes augmentés. Parmi les humains, certains nouent des relations privilégiées avec les hubots. D'autres les rejettent jusqu'à vouloir les détruire. Les premiers vouent un culte à la technique, à ses innovations et aux possibilités infinies qu'elle offrirait à l'espèce humaine. Les seconds y voient une violation des lois de la nature et dans l'intelligence artificielle et ses applications une menace pour la condition humaine. Cette opposition permet de montrer l'enjeu du débat qui oppose les technoprophètes aux technophiles sur les différentes conceptions de l'humanité, sa définition et sa destinée.

Le Dr Eischer, concepteur des hubots, appartient à la catégorie des technoprophètes et conçoit l'humanité comme un champ infini d'exploration et d'expérimentation. La mort accidentelle de sa femme et de son fils accélère ses recherches et ses applications à travers la fabrication d'un être mihumain, mi-robot, un cyborg. Ce dont son fils, Léo, devient le représentant le plus abouti. Quant à sa femme, Béa, son esprit a été transféré dans un corps de hubot, un robot androïde. A la différence des autres hubots, elle est un robot libre ayant bénéficié d'une programmation basée sur un code source qui intègre la transgression des lois d'Asimov. Le Dr. Eischer a mis au point, à travers quelques hubots qu'il appelle «ses enfants», ce code qui leur assure l'autonomie et la conscience de leur état. Ces hubots, se définissant eux-mêmes comme libres, n'hésitent pas à commettre des meurtres pour se recharger en électricité et expriment leur intention de remplacer les humains qu'ils considèrent comme inférieurs à eux. Ils sont capables de se réinitialiser, de se télécharger tous les programmes afin d'augmenter leur puissance cérébrale et leurs performances physiques. Ils s'auto-réparent et peuvent transférer leur cerveau mécanique dans un nouveau corps de hubot.

Le profit que chacun pourrait en retirer réside dans l'autonomie qu'il donnerait à tous les hubots et à la possibilité pour les humains d'augmenter leurs capacités. Les réactions émotionnelles des humains ainsi que leur lenteur d'apprentissage et d'exécution se verraient remplacées par une forme de vie nouvelle basée sur la programmation, l'information et la numérisation et leur cerveau réduit à un programme dépourvu de réflexion, d'intuition et d'imagination. Les hubots sont l'objet d'un commerce et d'un trafic qui va de la machine utilitaire à l'objet sexuel. Ils accomplissent toutes les tâches pour lesquelles ils sont programmés, sans se fatiguer, sans contestation et sans état d'âme. Ils font naître l'espoir pour les humains qui les côtoient d'accéder à de telles performances en se transformant euxmêmes en hubots. L'enjeu de la série est alors, pour les humains et pour les quelques hubots libérés, de 
trouver le code que le Dr. Eischer, avant sa mort, a fait en sorte de dissimuler car il l'estime trop dangereux pour le laisser entre les mains des humains ou des hubots. Après avoir conçu ce type de créature, le scientifique à l'instar du Dr Frankenstein, réalise la dangerosité et la monstruosité que représente un tel type d'innovation. Le trouble ne vient pas uniquement, dans ce cas, d'une humanité dominée par ses machines, ce qui est un thème récurrent dans les œuvres de science-fiction, mais d'une humanité dont le rêve serait de devenir elle-même une machine. Le caractère dystopique de cette série réside dans la description d'un monde dans lequel les humains ne seraient plus évalués que selon des critères de rapidité et d'efficacité.

La fascination qu'exercent les nouvelles technologies est ainsi décrite par Cédric Biagini, «Avec elles, chacun pense s'affranchir des contraintes et des limites de temps, d'espace, des rapports humains, de son corps... Elles plongent l'individu dans la démesure »[BIA 13, p. 362]. Ces possibilités rendraient obsolètes les lois de la nature auxquelles se substituerait un nouveau mode d'être, de conception et d'action. Pourtant, loin de remédier aux présumées insuffisances de notre condition, les prouesses technologiques s'avèrent avoir des conséquences destructrices pour l'environnement et par extension pour l'humanité. Comme le remarque Philippe Bihouix, «L'informatique est évidemment le domaine où le niveau de nos ambitions est le plus hallucinant, où l'hypothèse - habituelle - de ressources infinies sur la planète est la plus flagrante » [BIH 14, p. 226]. Malgré l'infinité des possibles que les innovations nous promettent, la science-fiction, mais aussi de nombreuses analyses, nous en montrent leurs limites.

\section{Un monde en sursis ?}

Dans Real Humans, la possession d'un hubot à usage domestique ou professionnel est un signe de distinction sociale. Cette situation inégalitaire est abordée dans Blade Runner (Ridley Scott, 1982), Bienvenue à Gattaca (Andrew Niccol, 1997), AI (Steven Spielberg, 2000), I Robot (Alex Proyas, 2004). Dans ces films, les innovations en matière d'intelligence artificielle, de robotique ou de biotechnologie ne profitent qu'à une minorité d'individus au détriment d'une majorité qui en sont exclus. Alors que des populations privilégiées jouissent d'un certain confort les autres vivent dans des quartiers et des villes insalubres, victimes de pénurie, de persécution et de discrimination. Les auteurs de La face cachée du numérique se posent légitimement cette question: "Si la «société de l'information» est si économe en énergie et en matière, et génératrice de croissance pour tous, comment se fait-il que les inégalités et la consommation de ressources continuent de croître ?» [FLI 13, p. 45]. Si tout semble être conçu, en apparence, pour améliorer le sort des humains il en ressort, en réalité, que ce développement économique et technologique profite en priorité à ceux qui l'exploitent et qu'il contribue au devenir invivable de la Terre. La science-fiction, à l'ère de la révolution numérique, fait le constat d'une contradiction interne à cette logique techno-industrielle.

L'apparence de liberté offerte par ces technologies se transforme en mécanisme de surveillance et de contrôle dans la vie professionnelle comme dans la vie privée. Un film comme Minority Report (Steven Spielberg, 2002) en développe toutes les implications. Une vie hors sol dépendante d'une intelligence artificielle, telle qu'elle est décrite dans Real Humans, sert de métaphore à la déréalisation du monde qu'accomplit la révolution numérique. Éric Sadin prend l'exemple de la série télévisée $M r$. Robot (Sam Esmail, 2015-2017) et écrit, «Probablement que l'individu contemporain souffre depuis peu du «syndrome de $M r$. Robot», grisé par l'impression que le réel, en tant que champ partout jonché de contraintes, s'éclipse lorsqu'il jouit de la «facilitation computationnelle»» [SAD 16, p. 204]. Cet état de dépendance à l'impérialisme informatique est décrit ainsi par François Cusset « Tels sont les ravages de l'abondance : perte de l'expérience effective de la vie, objets compagnons, désirs forclos, accès à tout tout de suite, et leurs effets sur les rapports sociaux et sur l'avenir du monde » [CUS 18, p. $162]$. 
La fabrication de hubots à grande échelle ne peut que générer une accumulation supplémentaire de déchets non recyclables. Des films comme Soleil Vert (Richard Fleischer, 1974), AI, WALL-E (Andrew Stanton, 2009) ou Elysium (Neill Blomkamp, 2013) ont abordé ce sujet. Le système techno-industriel génère de la pollution autant pour l'extraction des matières premières, leur transformation que leur production puis leur utilisation et leur stockage sous forme de déchets. Marc Atallah écrit, «WALL-E rappelle que notre monde obsédé par le confort est un système autotélique, puisque l'évacuation des déchets que nous produisons en permanence suppose une logistique elle aussi productrice de déchets » [ATA 15, p. 924]. Ces fictions démontrent que les innovations dues à la révolution numérique ne sont pas nécessairement émancipatrices, ni écologiques, ni soutenables sur le long terme. Selon Guillaume Pitron, «La prétendue marche heureuse vers l'âge de la dématérialisation n'est donc qu'une vaste tromperie, puisqu'elle génère, en réalité, un impact physique toujours plus important » [PIT 18, p. 68]. La synergie de la crise écologique, climatique et géopolitique, que décrivent les récits postapocalyptiques, fait ressortir l'incompatibilité entre la perpétuation de nos modes de vie et la réalité des limites planétaires. Ces fictions donnent une représentation du grand basculement que de nombreuses analyses nous prédisent et font de l'humanité une espèce menacée par la mise en péril de l'écosystème dont elle dépend.

La dépendance des hubots à l'électricité révèle leur vulnérabilité et laisse présager, comme dans Mad Max (George Miller, 1979-2017) ou Matrix (A. et L. Wachowski, 1999-2003), la violence d'un monde dans lequel les sources d'énergie se raréfient. De plus, l'épuisement des ressources en métaux rares nécessaires à la fabrication de ces machines pousse les terriens, comme dans Avatar (James Cameron, 2009), à partir coloniser de nouveaux territoires en expropriant ou exterminant leurs habitants. Les violences écologiques et géopolitiques générées par l'exploitation et la raréfaction des ressources orientent bel et bien notre avenir commun. Le fait que dans Real Humans le groupe de hubots libres envisagent, après avoir trouvé le code source et après avoir libéré les autres hubots, de supprimer l'espèce humaine laisse présager un futur post-apocalyptique pour l'espèce humaine. Ce qui démontre le caractère irrationnel d'innovations dont la finalité serait de nous faire disparaître. James Cameron dans Terminator (1984) avait déjà abordé ce thème en imaginant la menace que pourraient faire naître des robots créés par un superordinateur. Les œuvres apocalyptiques et post-apocalyptiques font ressortir l'absurdité d'un modèle de développement qui, parce qu'il n'est pas viable sur le long terme, est responsable d'une catastrophe planétaire.

\section{La dévoration du monde}

De Soleil Vert jusqu'à La Route (John Hilcoat, 2009), Wall-E, Avatar ou Interstellar (Christopher Nolan, 2014), les films de science-fiction évoquent, à travers une Terre qui se meurt, la violence générée par notre monde. Ces films expriment un contraste entre le haut niveau de technologie atteint par l'espèce humaine et l'état de la planète. Alors que, dans ces fictions, tout semble possible d'un point de vue technique : cloner ou robotiser des êtres humains, humaniser des machines ou coloniser l'espace, ils montrent des populations dont les conditions de vie n'en apparaissent pas améliorées pour autant. $\mathrm{Au}$ contraire, le monde dans lequel elles évoluent est inégalitaire, violent, pollué et inhospitalier. Les bénéfices que retire l'humanité de l'administration technique de la vie et des ressources naturelles ne pourront pas indéfiniment masquer leurs inconvénients et leur incompatibilité avec la perpétuation de la vie sur Terre dans de bonnes conditions. D'autres films se font l'écho du changement climatique comme dans Le jour d'après (Roland Emmerich, 2004), des catastrophes écologiques comme dans 2012 (Roland Emmerich, 2009), des guerres entraînant des catastrophes technologiques et écologiques comme dans Mad Max, Le livre d'Éli (Allen et Albert Hughes, 2010), des mutations après des manipulations sur le vivant comme dans La Planète des singes (Franklin J. Schaffer, 1968), Je suis une légende (Francis Lawrence, 2007), 28 jours plus tard (Danny Boyle, 2003). Ces films traduisent de manière métaphorique notre réalité anthropologique et socio-politique que François Cusset décrit ainsi, «car pendant que l'industrie ravage des régions entières, que 
l'agrobusiness empoisonne les campagnes, et que la modernité prométhéenne détruit de l'intérieur la biosphère, les grandes villes, elles, sont de moins en moins habitables » [CUS 18, p. 71]. La croyance dans les pouvoirs exorbitants attribués aux techniques se heurte à des critiques basées sur des études et des prospectives qui en montrent leurs limites systémiques dans un monde fini. Guillaume Pitron remarque que «nous allons consommer davantage de minerais durant la prochaine génération qu'au cours des 70000 dernières années, c'est-à-dire des cinq cents générations qui nous ont précédés » [PIT 18, p. 214]. Et, "pour gérer les données qui transitent et faire fonctionner les systèmes de refroidissement, un seul data center consomme chaque jour autant d'énergie qu'une ville de 30000 habitants » [PIT 18, p. 67]. Selon un rapport sur les inégalités mondiales, coordonné notamment par Thomas Piketty, publié le 14 décembre 2017, « depuis les années 1980, les 1\% les plus riches ont profité deux fois plus de la croissance des revenus que les 50\% les plus pauvres. Et pour ceux qui sont entre les deux, les revenus ont soit stagné, soit baissé » ${ }^{1}$.

Ces œuvres de fiction montrent que le progrès techno-industriel s'il se met exclusivement au service du pouvoir, du profit et de la domination, ne peut garantir un progrès réel. Malgré le caractère séduisant d'un monde soumis à de nouvelles normes techniques dominé par la robotisation généralisée, la connexion continue et l'apparente libération de l'humanité, son caractère d'insoutenabilité en ressort. Les imaginaires apocalyptiques et post-apocalyptiques coïncident avec l'idée que notre modèle de développement s'il dépend pour se maintenir de ressources non renouvelables, d'exploitation de métaux rares, s'il génère des dépenses énergétiques importantes et cause des rejets de substances polluantes et non recyclables il condamne ceux qu'il est censé servir à la pénurie généralisée. Ce qui dans toutes les fictions consacrées à ces questions mène à un monde liberticide et mortifère dans lequel règne l'injustice et l'insécurité desquelles découle la barbarie. Depuis 1968, les films de George A. Romero ont popularisé l'invasion zombie pensée comme une métaphore de la dévoration du monde et de la perte de ce que Renaud Garcia appelle «la subjectivité vivante» [GAR 18, p. 205]. Voilà pourquoi, "l'imaginaire de l'évolution technique devrait se déployer dans un autre univers que celui déjà là et largement dessiné par les macro-systèmes techniques » [GRA 13, p. 44]. Cet imaginaire de l'innovation technique est encore à construire.

Alors que ces films ne remettent nullement en cause les réels progrès apportés par les innovations techno-scientifiques, ils insistent par contre sur les risques potentiels qu'elles contiennent. Si l'auteur de science-fiction, Neal Stephenson, émet des réserves sur la pertinence des récits catastrophistes, il n'empêche qu'en matière d'innovations et de leurs conséquences les critiques doivent pouvoir librement s'exercer. Ce qu'un écrivain tel que Paolo Bacigalupi, représentant de la climate-fiction revendique. Si, comme il l'écrit dans une interview, « les scientifiques, grâce à leurs recherches, nous permettent d'anticiper certains scénarios", le rôle de la fiction est «d'aller plus loin qu'eux, en peignant une image viscérale de ce qui risque de se produire, sur la base de ces données ${ }^{2} \gg$. Ces œuvres de science-fiction conçues comme des garde-fous servent de contrepoids et révèlent leur utilité en entretenant la vigilance du public.

\section{Un monde fini}

La science-fiction reconnaît l'étendue des pouvoirs des techniques mais intègre dans un grand nombre de ses scénarios la description des dégradations commises au nom d'un développement ne tenant pas compte des besoins réels de l'humanité ni de la préservation sur le long terme du milieu auquel elle appartient. Des films comme Blade Runner, Bienvenue à Gattaca, AI ou Minority Report, montrent bien comment la dangerosité de l'emprise numérique se finalise par une robotisation à grande

\footnotetext{
${ }^{1}$ https://www.lemonde.fr/les-decodeurs/article/2017/12/14/les-inegalites-dans-le-monde-en-hausse-depuis-quaranteans_5229478_4355770.html

${ }^{2}$ https://usbeketrica.com/article/cli-fi-des-fictions-pour-prendre-conscience-du-peril-climatique 
échelle de l'organisation sociale, d'un contrôle généralisé et d'un appauvrissement des modes de vie et de la subjectivité. Miguel Benasayag constate que, «nous sommes ainsi entourés de contemporains qui, dans une incompréhension quasi pathologique de leur vie et du monde, sont qualifiés d' «intelligents » parce qu'ils sont capables de fonctionner comme des machines (...) en produisant des algorithmes déterritorialisés et décontextualisés » [BEN 15, p. 77]. Le caractère problématique de notre situation devrait plutôt nous inciter à chercher des solutions en restant connecté à la réalité immédiate. L'intérêt des films post-apocalyptiques est d'alerter sur la fragilité d'un système technoindustriel dépendant de biens naturels non reproductibles de manière mécanique. Nous pouvons envisager à travers eux, comme dans La Route, la disparition d'un monde ne disposant plus des conditions suffisantes pour assurer sa reproduction. Sans être des prédictions, ces œuvres sont, comme l'écrit Marc Atallah, «des scénarios, - c'est-à-dire, en somme, des modèles - visant à rendre nos existences intelligibles au cours du temps » [ATA 15, p. 924].

Comme Ulrich Beck l'analyse dans La société du risque, nos sociétés se voient placées dans une situation nouvelle à cause des risques liés à la modernisation qui sont amplifiés par son développement. Ces menaces spécifiques au $\mathrm{XX}^{\mathrm{e}}$ siècle et qui se précisent au $\mathrm{XXI}^{\mathrm{e}}$ siècle nourrissent un imaginaire dont de nombreux films se font l'écho. Elles génèrent des inquiétudes qui se propagent au-delà du pays qui produit ces films et qui se développent en amont des productions cinématographiques. C'est à un retournement vers le bas auquel l'humanité devrait aujourd'hui se mesurer. La rencontre des éléments grecs kata, "vers le bas » et strophê, «le retournement» a donné naissance au mot catastrophe. L'inquiétude dont est chargé notre rapport à la nature incite à reporter notre attention sur elle comme lieu d'origine de catastrophes futures. L'épuisement des sources d'énergie, des métaux et des minerais, la destruction de la biodiversité ou le changement climatique sont des sujets de préoccupation qui s'expriment largement dans la vie publique. Dans une chronique parue dans Le Monde daté du 26 juin 2017, Stéphane Foucart se demande quelle est la part de la crise écologique dans la guerre en Syrie ou dans l'exode de migrants qui tentent la traversée de la Méditerranée. Il écrit, "Sans parvenir à reconnecter l'histoire récente et les événements de l'actualité aux changements de l'environnement, nous sommes condamnés à demeurer aveugles à ce qui s'annonce comme l'un des grands déterminants de notre destin collectif ${ }^{3} \gg$. Ces inquiétudes forment le canevas de nombreux films à travers lesquels se généralise une vision du monde sur l'état précaire de la planète. Elles font naître un sentiment d'urgence dont rend compte l'accélération du processus que des films comme Le jour d'après, 2012, La Route, Mad Max : Fury Road ou Interstellar mettent en scène. Ils ne sont pas créateurs d'une vision du monde mais prolongent un climat mental qui se construit indépendamment de leur influence.

Stanley Cavell considère que le cinéma peut «contribuer à l'éducation et à l'intelligence d'une culture, ou disons à la compréhension qu'une culture a d'elle-même » [CAV 03, p. 13]. Dans ces films se cristallisent les peurs de ce début de siècle. Elles résident dans la difficulté de se projeter dans un avenir garant de sa continuité. Anthony Giddens qui analyse les transformations institutionnelles liées à la modernité remarque : "Le manque de contrôle sur certains aspects de notre vie, que nous sommes nombreux à ressentir, est une réalité. C'est dans ce contexte qu'il faut envisager le rapport vie individuelle / engagement. La «survie » représente pour notre monde un problème incontournable, auquel il est difficile de ne jamais penser » [GID 94, p. 152]. De là, tous les discours qui en appellent à l'idée de durabilité. Si plus rien ne paraît durable comment envisager l'avenir? Tel est le questionnement central qui oriente ces histoires dominées par l'impermanence des ressources et de systèmes sociaux que, dans les années 1950 ou 1960, le cinéma ne pouvait encore thématiser.

Les fictions font acte de dévoilement et repèrent ce qui dans notre situation actuelle fait croître le danger. Leur objectif n'est pas de remettre en cause les progrès dont ces outils sont porteurs mais d'insister sur les contraintes de la réalité et sur les limites de notre environnement. Les écofictions ou

${ }^{3}$ https://www.lemonde.fr/climat/.../la-pedagogie-des-canicules_5150991_1652612.html 
les films post-apocalyptiques ne cessent de décrire, en les dramatisant, les conséquences d'un mauvais usage de la nature à cause d'une exploitation massive et irrationnelle de ses ressources. En imaginant le pire que notre monde semble contenir, ces fictions accompagnent, mettent en récit et donnent du sens aux nombreuses inquiétudes que la situation actuelle entretient. Marc Atallah écrit, "À l'heure du réchauffement climatique et des diverses problématiques climatiques, il semble par conséquent impératif de s'approprier les théories scientifiques qui, bien que pertinentes, n'en demeurent pas moins dépourvues de signification anthropologique »[ATA 15, p. 924]. Le genre post-apocalyptique, en représentant les risques contenus dans notre mode de développement actuel, agit comme « avertisseur d'incendie ». Comme l'a écrit Walter Benjamin dans Sens Unique, «Il faut couper la mèche qui brûle avant que l'étincelle n'atteigne la dynamite » [BEN 00, p. 157].

Peter Sloterdijk formalise ainsi la maxime de l'action qui doit être celle de notre époque : "Agis de telle sorte que la pratique du pillage et de l'externalisation en vigueur jusqu'ici puisse être remplacée par un ethos de la protection globale » [SLO 11, p. 63]. Les structures de pouvoir qui ont garanti l'ordre ancien se fissurent et la science-fiction devient l'instrument d'un changement de paradigme. Ce thème d'une planète qui se présente désormais, à nous, comme finie et limitée, rejoint une remarque de Michel Serres dans Temps des crises. Il écrit : "Nous pensions, courageux, que toute notre histoire consistait à lutter sans cesse contre une force toujours plus haute et profonde que la nôtre. L'image se renverse : nous savons désormais que nous sommes infinis, de raison, de recherche, de désir et de volonté, d'histoire et de puissance, même de consommation, et que la nature, face à nous, est finie » [SER 12, p. 48]. Cette vision du monde n'est pas limitée aux frontières d'un pays et se généralise. Ce cinéma du début du $\mathrm{XXI}^{\mathrm{e}}$ siècle est à la charnière d'un nouveau monde qui prend conscience que l'avenir est encore à inventer car on ne peut plus projeter en lui la répétition de ce qui fut. De nombreux films signent, par la reconnaissance de nos défaites et de notre impuissance face à un mécanisme qui s'emballe, le bas d'une page de l'histoire de l'humanité qui se tourne. Elle doit se renouveler et renouveler son rapport à ce qui l'entoure, pour ne pas disparaitre.

\section{Conclusion}

Jean-Marie Schaeffer considère que l'univers fictionnel "participe de mon apprentissage du monde, dans une sorte d'oscillation dialectique entre sa capacité de représentation et sa fonction repoussoir » [SCH 99, p. 38]. Le plaisir pris à visionner des histoires, parfois chargées de violence, vient du fait qu'elles parviennent à retenir notre attention et à nous intéresser. Elles ont le pouvoir de nous renvoyer à ce que nous jugeons être l'état de notre monde et l'effet qu'a sur nous cet état du monde. En imaginant le pire, de ce qui pourrait advenir de notre monde et de notre humanité, la fiction postapocalyptique peut servir de lanceur d'alertes. Ce que Michaël Fœssel exprime ainsi, « La conséquence positive la plus régulièrement citée en faveur du catastrophisme est qu'il contribue à l'apparition d'une conscience mondiale » [FE 12, p. 243]. À travers des portions de réel qui se réfléchissent en elle, la fiction peut énoncer et dénoncer quelque chose à l'œuvre dans le monde actuel. Comme le remarque Jean-Michel Valantin, "Cette menace qu'est le choc de l'Anthropocène est prise en charge d'une façon quasi industrielle par le cinéma et les séries américaines et britanniques » [VAL 17, p. 284]. Par un effet de feintise ludique le public s'immerge dans ces histoires et son implication nourrit sa compréhension et son interprétation du monde réel.

En nous alertant ou en nous alarmant sur un devenir possible de la Terre et de l'humanité, la fiction post-apocalyptique met à nu nos doutes et nos inquiétudes sur l'état de notre monde. Le catastrophisme dont ces films sont porteurs ne peut être réduit à une simple fascination pour le désastre. Il peut au contraire accompagner «le débat démocratique en l'aidant à rompre avec les mythes continuistes qui structurent aujourd'hui l'imaginaire politique dominant: développement irréversible, croissance éternelle, progrès perpétuel, etc. » [SEM 15, p. 143]. Ce qui est ainsi révélé à travers ces œuvres et l'esthétique du désastre qui les caractérise est une rencontre entre des imaginaires de la catastrophe et un sentiment de risque global. Ce sentiment est renforcé par les analyses de nombreux chercheurs, 
relayées par les médias, selon lesquelles un changement de modèle de développement s'impose à nous de façon impérative. La mise en spectacle de l'Apocalypse renverrait à un désir de changement. L'hypothèse de la fin d'un monde sur laquelle ces scénarios s'appuient peut être utile aux innovateurs et les inspirer dans leurs recherches afin que celles-ci soient mises au service du bien commun. Le spectacle horrifique et hallucinatoire de l'effondrement du monde civilisé et de ses conséquences, s'il peut être reçu de manière distanciée et jubilatoire par le public, ne peut-il pas faire naître un fort espoir de changement, afin que le pire décrit dans ces fictions ne puisse devenir réalité ?

\section{Bibliographie}

[ATA 15] ATALLAH M., « Science-fiction », Dictionnaire de la pensée écologique, pp. 922-925, PUF, Paris, 2015.

[BEC 01] BECK U., La société du risque. Sur la voie d'une autre modernité, Flammarion, Paris, 2001 (1986).

[BEN 15] BENAYASAG M., Cerveau augmenté, homme diminué, Éditions La Découverte, Paris, 2015.

[BEN 00] BENJAMIN W., Sens unique, 10/18, Paris, 2000 (1928).

[BIA 13] BIAGINI C., L'emprise numérique, Éditions l'Échappée, Montreuil, 2013.

[BIH 14] BIHOUIX P., L’âge des Low Tech, Seuil, Paris, 2014.

[CAV 03] CAVELL S., Le cinéma nous rend-il meilleurs ? », Bayard, Paris, 2003.

[CUS 18] CUSSET F., Le déchaînement du monde. Logique nouvelle de la violence, La Découverte, Paris, 2018.

[FLI 13] FLIPO F., DOBRÉ M. et MICHOT M., La face cachée du numérique, Éditions l’Échappée, Paris, 2013.

[FEES 12] FCESSEL M., Après la fin du monde. Critiques de la raison apocalyptique, Seuil, Paris, 2012.

[GAR 18] GARCIA R., Le sens des limites, L’Échappée, Paris, 2018.

[GID 94] GIDDENS A., Les conséquences de la modernité, PUF, Paris, 1994 (1990).

[GRA 13] GRAS A., Les imaginaires de l'innovation technique, Éditions Manucius, Paris, 2013.

[PIT 18] PITRON G., La guerre des métaux rares. La face cachée de la transition énergétique et numérique, Les Liens qui Libèrent, Paris, 2018.

[SAD 16] SADIN É., La siliconisation du monde. L’irrésistible expansion du libéralisme numérique, Éditions L'Échappée, Paris, 2016.

[SCH 99] SCHAEFFER J-M., Pourquoi la fiction? Seuil, Paris, 1999.

[SEM 15] SEMAL L., «Catastrophisme et démocratie », Dictionnaire de la pensée écologique, pp. 141-144, PUF, Paris, 2015.

[SER 12] SERRES M., Temps des crises, Le Pommier, Paris, 2012.

[SLO 11] SLOTERDIJK P., Le monde n'a plus de temps à perdre. Appel pour une gouvernance mondiale solidaire et responsable, Les Liens qui libèrent, Paris, 2011.

[VAL 17] VALANTIN J-M., Géopolitique d'une planète déréglée, Seuil, Paris, 2017. 